\title{
ON THE BIFURCATION THEORY OF SEMILINEAR ELLIPTIC EIGENVALUE PROBLEMS
}

\author{
CHARLES V. COFFMAN ${ }^{1}$
}

\begin{abstract}
A standard "bootstrap" method is used to show that the bifurcation problem for the semilinear eigenvalue problem $\Delta u+\lambda f(x, u)=0$ in $\Omega,\left.u\right|_{\partial \Omega}=0$, where $f(x, 0) \equiv 0$, and $(\partial / \partial u) f(x, 0)>0$, and when formulated in terms of weak solutions, is a local problem, i.e. independent of the behavior of $f$ for large $u$. A principle of linearization for this problem is proved under mild differentiability conditions on $f$.
\end{abstract}

1. This note is concerned with the bifurcation problem for the semilinear elliptic eigenvalue problem

$$
-\Delta u=\lambda(P(x) u+g(x, u)), \quad x \in \Omega,\left.\quad u\right|_{\partial \Omega}=0 .
$$

Here $\Omega$ is a bounded region in $R^{N}(N \geqq 2)$ for which the Dirichlet problem is solvable, and $g$, defined for small $u$, is odd and monotone in $u$. For recent contributions to bifurcation theory for nonlinear elliptic eigenvalue problems and for additional references see [5].

The object here is twofold, namely to show that in the variational treatment of the bifurcation problem for (1) a polynomial growth condition on $g$, as usually required, is unnecessary and using this fact and results of [3] and/or [6], to derive bifurcation theorems for (1).

For simplicity we have restricted the linear operator on the left in (1) to be the Laplacian. Without difficulty the results obtained below can be extended to the larger class of real linear formally selfadjoint operators considered in [2]. The machinery for doing this is set up in [2].

2. The approach here to the eigenvalue problem (1) is through the study of the integral equation

$$
u(x)=\lambda \int_{\Omega} G(x, t) f(t, u(t)) d t,
$$

Received by the editors September 21, 1970 and, in revised form, April 9, 1971.

AMS 1970 subject classifications. Primary 35G30, 35J20, 35J25.

Key words and phrases. Bifurcation theory, bifurcation point, principle of linearization, "bootstrap" method, integral equation.

1 This research was supported by NSF grant GP-21512.

(c) American Mathematical Society 1972 
where $f(x, u)=P(x) u+g(x, u)$ and $G$ is the Green's function for

$$
-\Delta w=\rho \text { in } \Omega,\left.\quad w\right|_{\partial \Omega}=0 .
$$

To obtain certain required variational inequalities one must consider (2) in $L^{p}(\Omega)$, for an appropriate $p>2$, and for this it is necessary to assume that $f$ is defined on $\Omega \times R$ and satisfies a polynomial growth condition in $u$. It can be shown however, by a standard "bootstrap argument", that for appropriate $p$ and suitably restricted $f$, an $L^{p}$-eigenfunction of (2) agrees almost everywhere with a classical solution of (1), i.e. a function $u \in C^{2}(\Omega) \cap C(\bar{\Omega})$ and satisfying (1). This can be done, for example, as indicated in the proof of Theorem 4 in [1]. By similar arguments one can prove the following result which implies the superfluity of the polynomial growth condition in the bifurcation theory of (1).

THEOREM 1. Let $\Omega$ be a bounded region in $R^{N}(N \geqq 2)$ and assume that the problem (3) has a Green's function $G(x, t)$. Let $f(x, u)$ be continuous on $\bar{\Omega} \times R$ and satisfy $f(x, 0) \equiv 0$, and

$$
|f(x, u)| \leqq K\left(1+|u|^{\gamma}\right), \quad(x, u) \in \Omega \times R,
$$

for some $K>0, \gamma \geqq 0$.

If $p>\min \left(1, \frac{1}{2} N(\gamma-1)\right)$ and $\left(\lambda_{n}, u_{n}\right)$ is a sequence of $L^{p}$-solutions of (2) such that

and

$$
\left\|u_{n}\right\|_{p}=\left(\int_{\Omega}\left|u_{n}\right|^{p} d x\right)^{1 / p} \rightarrow 0 \text { as } n \rightarrow \infty,
$$

then

$$
\lambda_{n} \leqq \Lambda<\infty, \quad n=1,2, \cdots,
$$

$$
\left\|u_{n}\right\|_{\infty}=\underset{\Omega}{\operatorname{ess} \sup }\left|u_{n}(x)\right| \rightarrow 0 \quad \text { as } n \rightarrow \infty .
$$

The proof of Theorem 1 will require the following result.

\section{LEMMA 1. The integral operator}

$$
y \rightarrow \int_{\Omega} G(\cdot, t) y(t) d t
$$

is completely continuous from $L^{r_{1}}(\Omega)$ to $L^{s}(\Omega)$, for $1 \leqq r_{1}, s \leqq \infty$, provided $s^{-1}>r_{1}^{-1}-2 N^{-1}$.

Proof. From the definition of the Green's function for (3) and the maximum principle for harmonic functions there follows, for $x, t \in \Omega$,

$$
\begin{aligned}
|G(x, t)| & \leqq \text { const }(1+|\log | x-t||), & & N=2, \\
& \leqq \text { const }|x-t|^{-N+2}, & & N>2,
\end{aligned}
$$


and this implies, since $\Omega$ is bounded,

$$
\sup _{x \in \Omega} \int_{\Omega}|G(x, t)|^{a} d t<\infty,
$$

for $1 \leqq a<N /(N-2)$. This, together with the symmetry of $G$ implies the assertion of the lemma; see Theorem 9.5.6 [4, p. 658].

ProOF OF THEOREM 1. In view of the conditions on $f$, Theorem 19.1 of [8] implies that $u \rightarrow f(\cdot, u(\cdot))$ is continuous from $L^{r}(\Omega)$ to $L^{r_{1}}(\Omega)$ for finite $r_{1}$ with $1 \leqq r_{1} \leqq r / \gamma$. Combined with Lemma 1 , this implies that the operator

$$
u \rightarrow \int_{\Omega} K(\cdot, t) f(t, u(t)) d t
$$

is continuous from $L^{r}(\Omega)$ to $L^{s}(\Omega)$ for $1 \leqq r, s \leqq \infty$ and $s^{-1}>\gamma r^{-1}-2 N^{-1}$. In particular this operator is continuous from $L^{r}(\Omega)$ to $L^{\infty}(\Omega)$ for $r>\frac{1}{2} \gamma N$, and from $L^{r}(\Omega)$ to $L^{r+\delta}(\Omega)$ for $r \geqq p>\frac{1}{2} N(\gamma-1)$, where

$$
\delta=p(2 p-(\gamma-1) N) /(\gamma N-2 p)>0
$$

if $p \leqq r<\frac{1}{2} \gamma N$. Thus if

$$
v_{n}(x)=\int_{\Omega} G(x, t) f\left(t, u_{n}(t)\right) d t
$$

then from (5) it follows that

$$
\lim _{n \rightarrow \infty}\left(\int_{\Omega}\left|v_{n}\right|^{r} d x\right)^{1 / r}=0
$$

for $r=p+\delta$, and hence since $u_{n}=\lambda_{n} v_{n}$, from (6), it follows that

$$
\lim _{n \rightarrow \infty}\left(\int_{\Omega}\left|u_{n}\right|^{r} d x\right)^{1 / r}=0 .
$$

Repeating this argument with $p$ replaced by $r=p+\delta$ we conclude that $u_{n}$ tends to zero in the $L^{p+2 \delta}(\Omega)$-norm, and proceeding by induction, we conclude that $\left(7^{\prime}\right)$ holds for every finite $r \geqq p$. Finally, using the continuity of the nonlinear integral operator from $L^{r}(\Omega)$ to $L^{\infty}(\Omega)$ for $r>\frac{1}{2} \gamma N$, and arguing once again as above, we conclude that (7) holds. This completes the proof of Theorem 1 .

3. Theorem 1, combined with the results of [3], yields the following.

THEOREM 2. Suppose that $P(x)$ is bounded, positive, and locally Hölder continuous in $\Omega$ and that $g(x, u)$ is bounded and locally Hölder 
continuous in $(x, u)$ on $\{(x, u): x \in \Omega,|u|<c\}$, where $\Omega$ is as in Theorem 1 . Suppose in addition that $g(x, u)$ is odd

and monotone

$$
g(x, u)=-g(x,-u), \quad x \in \Omega, \quad|u|<c,
$$

$$
g\left(x, u_{1}\right) \geqq g\left(x, u_{2}\right), \quad x \in \Omega, \quad c>u_{1}>u_{2}>-c,
$$

and finally that

$$
g(x, u)=o(|u|), \quad \text { as } u \rightarrow 0,
$$

uniformly with respect to $x \in \Omega$.

Then every eigenvalue $\mu_{k}$ of the linear eigenvalue problem

$$
-\Delta u=\mu P(x) u, \quad x \in \Omega,\left.\quad u\right|_{\delta \Omega}=0,
$$

is a bifurcation point of (1).

A more precise formulation of the conclusion of Theorem 2 is contained in the assertion $(*)$ below.

Using [6, Theorem 2.2, p. 332] or [8, Theorem 26.8, p. 251] we can obtain a variant of Theorem 2 in which the assumptions of oddness and monotonicity on $g$ are replaced by the assumption that $g$ has a bounded continuous partial derivative with respect to its second argument.

TheOREM 3. Let $P(x)$ and $\Omega$ be as in Theorem 2 and let $g(x, u)$ be locally Hölder continuous in $(x, u)$ on $\{(x, u): x \in \Omega,|u|<c\}$. Suppose in addition that on its domain of definition $g(x, u)$ has a bounded continuous partial derivative, $g_{u}(x, u)$, with respect to $u$, and that

$$
g(x, 0)=g_{u}(x, 0)=0, \quad x \in \Omega .
$$

Then the conclusion of Theorem 2 is valid.

REMARK. In [6], in fact, more is actually proved than is stated in Theorem 2.2, p. 332. The full result which is obtained there shows that an approximation argument can be used to weaken the differentiability condition on $g$ in Theorem 3 to: $g(x, u)=o(|u|)$ as $|u| \rightarrow 0$, uniformly with respect to $x$. Thus in particular Theorem 2 follows from the results of [6]. On the other hand, the results of [3] yield, under the hypothesis of Theorem 2, certain results not contained in [6] concerning multiplicity of small eigenfunctions of (1) corresponding to a multiple eigenvalue of (8).

In order to deduce Theorem 2 from the results of [3] we require the following.

LEMMA 2. Let $g_{1}(x, u)$ be bounded and continuous in $(x, u)$ on $\Omega \times R$, and satisfy

$$
g_{1}(x, u)=o(|u|), \quad \text { as } u \rightarrow 0 \text {, }
$$


uniformly with respect to $x \in \Omega$. Then for $1 \leqq r<s<\infty, u \rightarrow g_{1}(x, u)$ is a continuous operator from $L^{s}(\Omega)$ to $L^{r}(\Omega)$, and is Fréchet differentiable at zero; its Fréchet derivative at zero is zero.

Proof. Since $g_{1}(x, u)$ is bounded and continuous the continuity follows at once from Theorem 19.1, [8]. If we set

$$
\begin{aligned}
h(x, u) & =0, & & u=0, \\
& =u^{-1} g_{1}(x, u), & & u \neq 0,
\end{aligned}
$$

then $h(x, u)$ is also bounded and continuous on $\Omega \times R$, thus, by the result just quoted, $u \rightarrow h(x, u)$ is continuous from $L^{s}(\Omega)$ to $L^{r^{\prime}}(\Omega)$, where $1 / r^{\prime}+1 / s=1 / r$. Since multiplication $(v, w) \rightarrow v \cdot w$, is continuous from $L^{s}(\Omega) \times L^{r^{\prime}}(\Omega)$ to $L^{r}(\Omega)$, the result follows.

Proof of THeORem 2. Clearly $g(x, u)$ has an extension $g_{1}(x, u)$ to $\Omega \times R$ which is odd and monotone and satisfies the hypothesis of Lemma 2 . Since $P$ is bounded and continuous, for any $r, s$ with $1 \leqq r<s<\infty$, the operator $u \rightarrow P(x) u+g_{1}(x, u)$ from $L^{s}(\Omega)$ to $L^{r}(\Omega)$ is continuous, and Fréchet differentiable at zero, with its Fréchet derivative at 0 being the operator $u \rightarrow P(x) u$.

We consider the integral equation

$$
u(x)=\lambda \int_{\Omega} G(x, t)\left(P(t) u(t)+g_{1}(t, u(t))\right) d t,
$$

in $L^{p}(\Omega)$ where

$$
2<p<2 N /(N-2)
$$

and regard the operator on the right in (9) as the composition of the Nemytsky operator $u \rightarrow P(t) u+g_{1}(t, u)$ from $L^{p}(\Omega)$ to $L^{q}(\Omega)$, and the integral operator $y \rightarrow \int_{\Omega} G(\cdot, t) y(t) d t$ from $L^{q}(\Omega)$ to $L^{p}(\Omega)$, where $1 / p+1 / q=1$. By virtue of Lemma 1 the inequality (10) insures the continuity of the integral operator and, since $q<2<p$, Lemma 2 implies continuity and Fréchet differentiability at zero of the Nemytsky operator. It follows that the principle of linearization $(\dagger)$ of [3] is applicable to the integral equation (9) in $L^{p}(\Omega)$. We conclude from the result just quoted that every eigenvalue of

$$
y(x)=\mu \int_{\Omega} G(x, t) P(t) y(t) d t,
$$

or equivalently, every eigenvalue of (8), is a bifurcation point of (9), considered in $L^{p}(\Omega)$. More specifically, if $\mu$ is an eigenvalue of (8) then for every $\epsilon>0$ there exist nontrivial $L^{p}$-solutions $(\lambda, u)$ of (9) with $|\lambda-\mu|<\epsilon,\|u\|_{p}<\epsilon$. Because of Theorem 1 this assertion remains 
valid when the $L^{p}$-norm is replaced by the $L^{\infty}$-norm (notice that in this case the constant $\gamma$ in Theorem 1 can be taken to be 0$)$. If $(\lambda, u)$ is a solution of (9) with $\|u\|_{\infty}<c$, then, because of the Hölder continuity of $P$ and $g$, it readily follows that $u$ is (up to difference on a set of measure zero) a solution of (1). Thus we have the following.

$\left.{ }^{*}\right)$ If $\mu$ is an eigenvalue of (8), then for every $\epsilon>0$ there exist nontrivial solutions $(\lambda, u)$ of (1) with $|\lambda-\mu|<\epsilon,\|u\|_{\infty}<\epsilon$.

ProOF of THEOREM 3. Take $g_{1}(x, u)$ to be an extension of $g(x, u)$ to $\Omega \times R$ which is continuous and bounded and which has a bounded continuous partial derivative $g_{1 u}(x, u)$ with respect to $u$. (Actually $g(x, u)$ may not have such an extension but its restriction to $\left\{(x, u): x \in \Omega,|u|<c^{\prime}\right\}$ for $0<c^{\prime}<c$ will; clearly there is no loss of generality in first taking such a restriction of $g$.) It follows from [8, Theorem 19.1, p. 154] that $u \rightarrow g_{1}(\cdot, u(\cdot))$ and $u \rightarrow g_{1 u}(\cdot, u(\cdot))$ are continuous from $L^{r}(\Omega)$ to $L^{s}(\Omega)$ for $1 \leqq r, s<\infty$. It then follows from [6, Theorem 2.2, p. 332] that every eigenvalue of (11) is a bifurcation point of (9) in $L^{p}(\Omega)$, where $p$ satisfies (10). (The application of the result just quoted to (9) is given in [8, Theorem 26.8 , p. 251], to which the reader is referred for details; the relevant theorem from [6] is quoted as [8, Theorem 17.7, p. 146].) The conclusion $\left({ }^{*}\right)$ now follows just as previously in the proof of Theorem 2.

REMARK. It is interesting to note that while Theorem 2 insures the existence of eigenfunctions of (1) when $P$ is positive, regardless of the behavior of $g$ for large $u$, (1) can fail to have any eigenfunctions when $P \equiv 0$. Such an example is due to Pohozaev [7]; consider the problem

$$
\begin{aligned}
\Delta u+\lambda|u|^{m} \operatorname{sgn} u & =0, & & |x|<1, \\
u(x) & =0, & & |x|=1 .
\end{aligned}
$$

An eigenfunction $u$ of this problem, corresponding to the eigenvalue $\lambda$, must satisfy [7]

$$
\lambda(N /(m+1)-(N-2) / 2) \int_{|x| \leqq 1}|u|^{m+1} d x=\int_{|x|=1} u_{v}^{2} d s,
$$

where $d s$ is the differential of surface area and $u_{v}$ is the outward normal derivative of $u$. One readily sees from this that the problem has eigenfunctions only if $m<(N+2) /(N-2)$, (by the maximum principle an eigenvalue of this problem must be positive).

\section{REFERENCES}

1. C. V. Coffman, An existence theorem for a class of non-linear integral equations with applications to a non-linear elliptic boundary value problem, J. Math. Mech. 18 (1968/69), 411-421. MR 41 \#2330.

2. - On a class of non-linear elliptic boundary value problems, J. Math. Mech. 19 (1969/70), 351-356. MR 39 \#7280. 
3. C. V. Coffman, Spectral theory of monotone Hammerstein operators, Pacific J. Math. 36 (1971), 303-322.

4. R. E. Edwards, Functional analysis. Theory and applications, Holt, Rinehart and Winston, New York, 1965. MR 36 \#4308.

5. J. B. Keller and S. Antman (Editors), Bifurcation theory and nonlinear eigenvalue problems, Benjamin, New York, 1969. MR 39 \#2555.

6. M. A. Krasnosel'skiî, Topological methods in the theory of nonlinear integral equations, GITTL, Moscow, 1956; English transl., Macmillan, New York, 1964. MR 20 \#3464; MR 28 \#2414.

7. S. I. Pohožaev, On the eigenfunctions of the equation $\Delta u+\lambda f(u)=0$, Dokl. Akad. Nauk SSSR 165 (1965), 36-39 = Soviet Math. Dokl. 6 (1965), 1408-1411. MR 33 \#411.

8. M. M. Vainberg, Variational methods for the study of nonlinear operators, HoldenDay, San Francisco, Calif., 1964. MR 31 \#638.

Department of Mathematics, Carnegie-Mellon University, Pittsburgh, PenNSylvania 15213 